\title{
The Barriers to Health Promotion Activities through Perception of Women with Chronic Disease
}

\author{
Omaima Mohamed Elalem ${ }^{1, *}$, Magda Aly Mohamed ${ }^{1}$, Soheir Tawfeek Ahamed ${ }^{2}$ \\ ${ }^{1}$ Community \& Family Health Nursing Department, Faculty of Nursing, Port-said University, Egypt \\ ${ }^{2}$ Medical Surgical Nursing Department, Faculty of Nursing/ Ain Shams University, Egypt \\ *Corresponding author: oelalem15@gmail.com
}

\begin{abstract}
Background: One of the greatest challenges that will face health systems globally in the twenty-first century will be the increasing burden of chronic diseases. Chronic diseases are one of the most important barriers that have a direct and negative impact on health promotion for women. Aim of this study was to identify the barriers to health promotion activities for women with chronic disease. Subjects and methods: A cross-sectional study design was utilized to achieve the aim of the current study. Setting: This study was conducted in primary health care centers and outpatient clinics of governmental hospital located in Port-Said City. Subjects: Purposive samples of 744 adult women who surfing from chronic disease. Tools of data collections: two tools were used to collect data. Tool I: A structured questionnaire sheet. Tool II: Barriers to Health Promoting Activities for Disabled Persons Scale (BHADP) obesity was determined based on body mass index (BMI). Results the majority (79.4\%) of the study sample had high level barrier of health promotion. the five highest leading barriers items were: Embarrassment about my appearance; Lack of support from family, friends; Lack of help from health care professionals; Bad weather and; No one to help. 38.6\%) of women who have a high score of levels of Health Promotion Barriers' suffering of Diabetes Mellitus. There was a strong significant correlation between the levels of Health Promotion Barriers' and body mass index, level of education and duration of chronic disease with p-value $\leq 0.001$. This study concludes that women with chronic diseases had a high score of health promotion barrier. The study recommends a greater emphasis on assessing and managing perceived barriers to health promotion activities in health education and policy development and proposes a conceptual model for understanding perceived barriers to action.
\end{abstract}

Keywords: Barriers to Health Promotion, Chronic Disease, Factors

Cite This Article: Omaima Mohamed Elalem, Magda Aly Mohamed, and Soheir Tawfeek Ahamed, "The Barriers to Health Promotion Activities through Perception of Women with Chronic Disease." American Journal of Nursing Research, vol. 6, no. 2 (2018): 229-236. doi: 10.12691/ajnr-6-5-3.

\section{Introduction}

One of the greatest challenges that will face health systems globally in the twenty-first century will be the increasing burden of chronic diseases, as a result of improved living conditions, better prevention, infectious diseases management, medical technological improvements and overall aging of the population. [1] An increasing number of people who are living with chronic diseases that can adversely affect Health-related quality of life (HRQOL). This is because chronic diseases are slow to improve and require long medical treatment that may limit the productivity of patients and their ability to live well. Among these diseases include cancer, heart disease, stroke, diabetes, and HIV and kidney disease. [2]

The US National Center for Health Statistics has identified a long-term chronic disease (3 months or more). [3] Chronic diseases cannot generally be prevented by vaccines or treated with drugs, nor do they disappear. In Egypt, there are no accurate statistics on the incidence of chronic diseases, but it was noted that it is more common with age and the most important cause of death, as $82 \%$ of the deaths were suffering from chronic diseases. [4,5]

There are known risk factors associated with chronic diseases. Some of these risk factors such as age, gender, family history, ethnicity and genetic factors cannot be modified. Other risk factors often referred to as lifestyle risk factors that can be modifiable by improving health promotion for the patient with chronic diseases, such risk factors are smoking, nutrition, alcohol, physical activity, and obesity. [6] In addition, the long-term effects of chronic diseases (complications) are among the most important obstacles to improving health, because they directly affect the patient's physical and financial ability. [7] Hence, the important role of health promotion as a second line defense to prevent the complications of chronic disease. [8]

Health promotion has become a popular issue in the fields of medicine, community health, and nursing. [9] As a result of the "Health for All" appeal by the World Health Organization, many countries have adjusted their medical health care systems to include health promotion in addition to treating disease. Such countries have cited health promotion as an important issue. [10]

Health promotion is directed toward increasing the individual's level of well-being and self-actualization. It 
focuses on the efforts to approach or move toward a positively valence state of high-level health and wellbeing. It may be true that for some health behaviors, both approaching a positive state (i.e.; working toward control of chronic disease) and avoiding a negative state, as in the protection-motivation theory (i.e.; preventing complications of long term uncontrolled chronic diseases), may serve as motivators for adopting health behaviors. [11]

The health status of female with chronic diseases is a global concern. In Egyptian society, the female faces many barriers that affect women's health, such as the nature of the diet, poor economic conditions, and the nature of culture that contributes to the neglect of women's health care. Women with chronic diseases also suffer from high mortality and disability rates compared with men. [12] Limited access to health promotion, disease prevention and health care programs contributes to these health challenges. In accessing health services in Arab countries these barriers can be social and cultural, administrative and organizational or financial and may impact on gender and ethnic groups to different degrees. [13]

Recent studies indicate that lack of finance, lack of attention by the family of the women's with chronic diseases, lack of awareness and needs of educational programs were the most important barriers that directly insisted on the levels of health promotion activities, both in outpatient and health centers. [14] Success in facilitating health promotion programs in clinics or health centers will require a change in the environment, especially those of the health team as well as women with chronic diseases. A combination of changing attitudes and the availability of an enthusiastic person (such as a nurse promoting health) may be needed to lead activities in order to achieve an increase in the level of health promotion among women with chronic diseases. [15]

The role of nurses has included clinical nursing practices, consultation, follow-up treatment, patient education and illness prevention. This has improved the availability of health-care services, reduced symptoms of chronic diseases, increased cost-effectiveness and enhanced customers' experiences of health-care services. [15] In addition, health promotion by nurses can lead to many positive health outcomes including adherence, quality of life, and patients' knowledge of their illness and selfmanagement which contributes to the decreases of the chronic diseases complications. [16,17,18]

\subsection{Significance of the Study}

Despite the importance of health promotion as a second level of prevention, there are no studies that have paid attention to the barriers that affect health promotion for women's with chronic diseases. Researchers therefore hope that this study will help to understand the perceptions of women with chronic disease about the barriers to health promotion lifestyle activities. And provide some evidence of a difference in women's perceptions and argue that these differences may have significant implications for improving health promotion for women's with chronic diseases in future.

Aim of the study: This study aims to identify the barriers to health promotion activities for women with chronic disease.

\subsection{Research Question}

1. What is the level of barriers to health promotion activities through perception of women with chronic disease?

2. What is the ranking of barriers for health promotion activities among women with chronic diseases?

3. What is the relation between the types of chronic diseases and level of health promotion barriers?

\section{Subject and Methods}

\section{Research Design}

A cross-sectional study design was utilized to achieve the aim of the current study.

\section{Study Setting}

This study was conducted in primary health care centers and outpatient clinics of governmental hospital located in Port-Said City which includes primary health care centers of El-Monakh, El-Kuwait, EL-arab, Osman Ebn Affan and Omar Ebn El-Kattab and Outpatient Clinics of El-Amery, Elnaser, El-Zhour and Port-Fouad hospitals.

\section{Subjects}

Purposive samples of 744 adult women who attend the above-mentioned settings were selected and meet the inclusion criteria of (a) suffer from chronic diseases and are followed in outpatient clinic and health centers as (Diabetic, heart disease, hypertensive, rheumatoid arthritis, COPD and thyroid disturbance) and excluded the critical patient. (b) Agree to participate in the study.

Tools of data collections: two tools were used by the researchers to collect data pertinent of this study.

Tool I: A structured questionnaire sheet was developed after review of the literature by the researchers to collect the following: it consists of; a) Demographic Data such as (age, education, Occupation and Types of chronic diseases; b) The Modified Risk Factors of the Women's such as Regular checkup, Diet, Exercise, Activities, obesity and Smoking.

* Obesity was determined based on body mass index (BMI) which is calculated in $\mathrm{kg} / \mathrm{m}^{2}$ according to the following equation: [19]

$$
\mathrm{BMI}=\frac{\text { Body weight in } \mathrm{kg}}{(\text { Height in meters })^{2}}
$$

BMI was categorized according to the World Health Organization criteria, where a BMI $<18.5 \mathrm{~kg} / \mathrm{m}^{2}$ is considered under-weight, a BMI ranged from (18.5 24.99) $\mathrm{kg} / \mathrm{m}^{2}$ is considered normal, a BMI between 25 and $29.99 \mathrm{~kg} / \mathrm{m}^{2}$ is considered overweight, and a BMI $\geq 30 \mathrm{~kg} / \mathrm{m}^{2}$ is considered obese.

Tool II: Barriers to Health Promoting Activities for Disabled Persons Scale (BHADP) (Becker \& Stuifbergen, 2004) The Barriers to Health Promoting Activities for Disabled Persons Scale is an 18-item, 4-point scale to measure individual barriers to take care of health. The original scale had 16 items; two additional barrier items were added in 1991 at the suggestion of people with disabilities. These items address the weather and lack of help from health care professionals. 
Respondents are asked to indicate how often listed barriers keep them from taking care of their health. Items include intrapersonal, interpersonal, and environmental barriers. Examples of barriers include being too tired, having other responsibilities, and lack of transportation. Responses are scored from 1 (never) to 4 (routinely). Scores range from 18-72. Higher scores indicate greater perceived barriers. [20] Statistically presentation of data was derived from that score for the total were evaluated as Higher Barrier $\geq 70 \%$ or lower Barrier $<70 \%$.

\subsection{Validity and Reliability of the Tool}

Barriers to Health Promoting Activities for Disabled Persons Scale (BHADP) was translated and face, content and concurrent validity were revised and ensured by five experts in different field of nursing at the faculty of nursing, Port Said University. A few changes were made for few wards. The developed and validated the BHADP was tested for reliability on a sample of $10 \%$ test retest result using alpha Chronbach revealed that all items are significantly differed and have a correlation coefficient above the threshold of significant $(r=.8)$.

\subsection{Pilot Study}

A pilot study was conducted after the development of the questionnaire and before starting the data collection on $10 \%$ of the study sample, to test the applicability, feasibility and to ensure easy understandability for ordinary women and to explore any unclear points of the study tool. It served to estimate the time needed to complete the questionnaire. The necessary modifications were done after obtaining the result of the pilot study and expertise opinions. Otherwise, the women included in the pilot were excluded from the study sample.

\subsection{Administrative Design}

A formal letter was issued from Dean of Faculty of Nursing in Port Said University. Then the study was approved by the directorate of primary health care centers and hospital outpatient clinics.

\subsection{Ethical Consideration}

The researchers fulfilled the official steps required to get the approval for carrying out the study. Informed verbal consent was obtained from all participants to participate in the study. The confidentiality and anonymity of any obtained data was ensuring through coding of all data. The purpose of the study was explained to each participant during personal interviews. The purpose of the study was explained to each woman during personal interviews.

\subsection{Field Work}

The researcher started by reviewing the related literature to gain more in-depth information about the subjects, and to be able to design the appropriate data collection tools. This took about two months. Then the developed tools were reviewed by experts in
Medical-surgical and community nursing. When the tools were finalized after pilot testing the actual field work started. After obtaining official permissions, the researcher started to explain the aim of the study to participant female patients and they were reassured that the information obtained is strictly confidential. The duration of data collection lasted seven months from February to end of August 2017. It took 20 to 25 minutes for each patient to collect the tools form.

\subsection{Statistical Design}

Data were analyzed using the Statistical Package for Social Sciences (SPSS), Version 20.0. A simple descriptive analysis in the form of percentages, arithmetic means, and standard deviations was used for data analysis. A chi-squared test was used to test the significance of the association between barrier of health promotion and demographic characteristics of women. Spearman coefficient test and Binary logistic regression was used considering the barrier of health promotion as the dependent variable. A P-value of $\leq 0.05$ was considered statistically significant.

\section{Results}

The study results revealed that more than two third of them $(44.8 \%)$ were in age group more 51 years. As regard to BMI the majority of the study sample (59.7\%) was overweight. In relation to educational level, the highest frequency was either illiterate or primary education (33.5\% or $34.7 \%$ ) respectively. As regards to occupation the highest frequency (75.8\%) were housewives. In regards to income, the highest frequency (70.2\%) was their income was not sufficient.

Table 1. Distribution of the study sample according the level of health promotion barriers $(n=744)$

\begin{tabular}{|l|c|c|}
\hline Barrier of health promotion & No. & $\mathbf{\%}$ \\
\hline Lower Barrier & 153 & 20.6 \\
\hline Higher Barrier & 591 & 79.4 \\
\hline Total score & \multicolumn{2}{|c|}{$32.0-69.0$} \\
\hline Min. - Max. & \multicolumn{2}{|c|}{$55.24 \pm 6.74$} \\
\hline Mean \pm SD. &
\end{tabular}

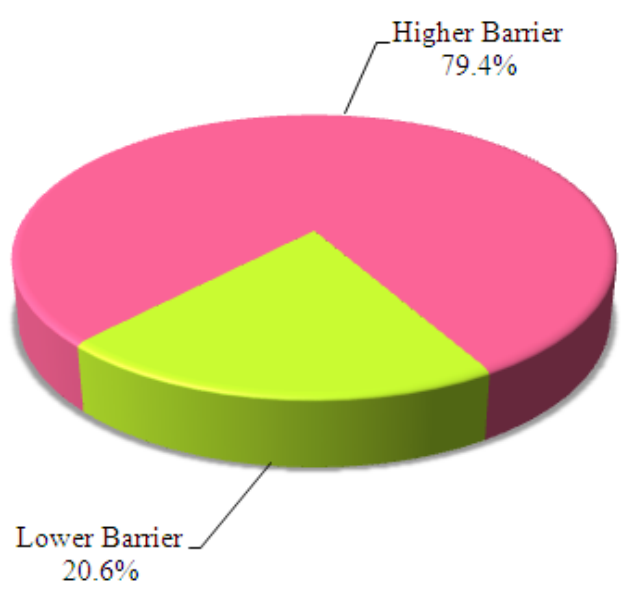

Figure 1. Distribution of the study sample according level of barrier of health promotion 
Table 1 shows that the majority (79.4\%) of the study sample had high level barriers' of health promotion. The mean and standard deviation of the level of Health Promotion Barriers was 55.24 \pm 6.74 .

Table 2 illustrating that the ranking of the first five major elements of barrier that have influenced health promotion and have been about embarrassment about appearance; lack of support from family and friends. Lack of assistance from health care professionals; bad weather and lack of assistance from others, while the five lowest barriers by ascending order were: Concern about safety, lack of transportation, overlapping responsibilities; low feeling of inability to do things correct.

3 elbaT shows that more one third (38.6\%) of the studied women who have a high score of levels of health promotion barriers suffering from diabetes mellitus followed by rheumatoid arthritis and hypertension (21.8\% and $20.8 \%$ ) respectively. A highly significant association was detected between the levels of health promotion barriers' and the women who suffer from diabetes, cardiovascular disease and rheumatoid arthritis. $\mathrm{p} \leq 0.05$.

4 elbaT revealed that there was a highly statistical significant difference between the levels of Health Promotion Barriers and body mass index, income and periodic medical checkup with $\mathrm{p}$-value $\leq 0.001$. While statistically significant association was detected between the levels of Health Promotion Barriers and age, occupation, level of education and Support from family/friends with $\mathrm{p} \leq 0.05$. On the other hand, there was no statistically significant association between the levels of Health Promotion Barriers' and following diet and doing exercise.

Table 5 clarified that there was a strong significant correlation between the levels of Health Promotion Barriers and body mass index, level of education and duration of chronic disease with $p$-value $\leq 0.001$. On the other hand, this table also revealed a significant negative association between the levels of Health Promotion Barriers and income and Women's impression of their health.

Table 6 shows a significant positive association between the levels of Health Promotion Barriers and body mass index, (odds ratio $[\mathrm{OR}]=2.127$ ); Types of chronic diseases $(\mathrm{OR}=1.135)$; duration of diseases $(\mathrm{OR}=1.271)$, while a significant negative association was detected between the levels of Health Promotion Barriers' and many factors like; age $(\mathrm{OR}=0.672)$; occupation $(\mathrm{OR}=$ $0.468)$; income $(\mathrm{OR}=0.277)$; level of education (OR $=0.861)$; Support from family/friends $(\mathrm{OR}=0.343)$; Women's impression of their health(OR $=0.789)$; and periodic medical checkup $(\mathrm{OR}=0.122)$.

Table 2. Rank of health promotion barriers Item Scores as perceived by studied women $(\mathbf{n}=\mathbf{7 4 4})$.

\begin{tabular}{|c|c|c|}
\hline Items & Sum item score & Rank \\
\hline Embarrassment about my appearance & 2838 & 1 \\
\hline Lack of support from family, friends & 2721 & 2 \\
\hline Lack of help from health care professionals & 2675 & 3 \\
\hline Bad weather & 2580 & 4 \\
\hline No one to help me & 2529 & 5 \\
\hline Not interested & 2484 & 6 \\
\hline Difficulty with communication & 2466 & 7 \\
\hline lack of convenient facilities & 2319 & 8 \\
\hline Too tired & 2220 & 9 \\
\hline Feeling what I do doesn't help & 2181 & 10 \\
\hline Lack of time & 2181 & 11 \\
\hline Lack of information about what to do & 2106 & 12 \\
\hline Lack of money & 2088 & 13 \\
\hline Feeling I can't do things correctly & 2043 & 14 \\
\hline Impairment & 2037 & 15 \\
\hline Interferes with other responsibilities & 1998 & 16 \\
\hline Lack of transportation & 1908 & 17 \\
\hline Concern about safety & 1686 & 18 \\
\hline
\end{tabular}

Table 3. Relation between level of barrier of health promotion and Types of chronic disease $(n=744)$

\begin{tabular}{|c|c|c|c|c|c|c|}
\hline \multicolumn{7}{|c|}{ Level of health promotion barrier } \\
\hline & \multicolumn{2}{|c|}{$\begin{array}{c}\text { Lower score } \\
(\mathrm{n}=153)\end{array}$} & \multicolumn{2}{|c|}{$\begin{array}{c}\text { Higher score } \\
(n=591)\end{array}$} & \multirow[t]{2}{*}{$\chi^{2}$} & \multirow{2}{*}{$\mathbf{p}$} \\
\hline & No. & $\%$ & No. & $\%$ & & \\
\hline \multicolumn{7}{|l|}{ Types of chronic disease } \\
\hline Diabetes & 42 & 27.5 & 228 & 38.6 & $6.509 *$ & $0.011 *$ \\
\hline Hypertension & 42 & 27.5 & 123 & 20.8 & 3.104 & 0.078 \\
\hline Cardiovascular disease & 33 & 21.5 & 54 & 9.1 & $18.189 *$ & $<0.001^{*}$ \\
\hline Thyroid & 11 & 7.2 & 30 & 5.1 & 1.043 & 0.307 \\
\hline Rheumatoid arthritis & 18 & 11.7 & 129 & 21.8 & $7.762 *$ & $0.005 *$ \\
\hline C. O.P. D & 7 & 4.6 & 27 & 4.6 & 0.00 & 1.000 \\
\hline
\end{tabular}

$\chi^{2}, \mathrm{p}: \chi^{2}$ and $\mathrm{p}$ values for Chi square test

MC: $p$ value for Monte Carlo for Chi square test

*: Statistically significant at $\mathrm{p} \leq 0.05$. 
Table 4. Relation between level of barrier of health promotion and different parameters $(n=744)$

\begin{tabular}{|c|c|c|c|c|c|c|}
\hline \multicolumn{7}{|c|}{ Level of health promotion barrier } \\
\hline & \multicolumn{2}{|c|}{$\begin{array}{l}\text { Lower score } \\
(\mathbf{n}=153)\end{array}$} & \multicolumn{2}{|c|}{$\begin{array}{c}\text { Higher score } \\
(\mathrm{n}=591)\end{array}$} & \multirow[t]{2}{*}{$\mathbf{X}^{2}$} & \multirow[t]{2}{*}{$\mathbf{P}$} \\
\hline & No. & $\%$ & No. & $\%$ & & \\
\hline \multicolumn{7}{|l|}{ Age } \\
\hline $20-30$ & 9 & 5.9 & 60 & 10.2 & \multirow{4}{*}{$8.781^{*}$} & \multirow{4}{*}{$0.032^{*}$} \\
\hline $31-40$ & 24 & 15.7 & 108 & 18.3 & & \\
\hline $41-50$ & 36 & 23.5 & 174 & 29.4 & & \\
\hline Over 51 & 84 & 54.9 & 249 & 42.1 & & \\
\hline \multicolumn{7}{|l|}{ BMI (kg/m2) } \\
\hline Normal & 37 & 24.2 & 95 & 16.1 & \multirow{3}{*}{$13.760^{*}$} & \multirow{3}{*}{$0.001^{*}$} \\
\hline Overweight & 97 & 63.4 & 347 & 58.7 & & \\
\hline Obese & 19 & 12.4 & 149 & 25.2 & & \\
\hline \multicolumn{7}{|l|}{ Occupation } \\
\hline Working & 21 & 13.7 & 147 & 24.9 & \multirow{3}{*}{$8.672^{*}$} & \multirow{3}{*}{$0.013^{*}$} \\
\hline Retired & 3 & 2.0 & 9 & 1.5 & & \\
\hline House wife & 129 & 84.3 & 435 & 73.6 & & \\
\hline \multicolumn{7}{|l|}{ Income } \\
\hline Sufficient & 21 & 13.7 & 201 & 34.0 & \multirow{2}{*}{$23.887^{*}$} & \multirow{2}{*}{$<0.001^{*}$} \\
\hline Not sufficient & 132 & 86.3 & 390 & 66.0 & & \\
\hline \multicolumn{7}{|l|}{ Level of education } \\
\hline Illiteracy & 66 & 43.1 & 183 & 31.0 & \multirow{5}{*}{$17.081^{*}$} & \multirow{5}{*}{$0.002^{*}$} \\
\hline Primary education & 51 & 33.3 & 207 & 35.0 & & \\
\hline Secondary education & 30 & 19.6 & 114 & 19.3 & & \\
\hline University & 6 & 3.9 & 78 & 13.2 & & \\
\hline Other & 0 & 0.0 & 9 & 1.5 & & \\
\hline \multicolumn{7}{|c|}{ Support from family/friends } \\
\hline Yes & 129 & 84.3 & 543 & 91.9 & \multirow{2}{*}{$7.956^{*}$} & \multirow{2}{*}{$0.005^{*}$} \\
\hline No & 24 & 15.7 & 48 & 8.1 & & \\
\hline \multicolumn{7}{|c|}{ Periodic medical checkup } \\
\hline Yes & 84 & 54.9 & 498 & 84.3 & \multirow{2}{*}{$61.516^{*}$} & \multirow{2}{*}{$<0.001^{*}$} \\
\hline No & 69 & 45.1 & 93 & 15.7 & & \\
\hline \multicolumn{7}{|l|}{ Following diet } \\
\hline Yes & 51 & 33.3 & 246 & 41.6 & $x^{2}-3483$ & م06? \\
\hline No & 102 & 66.7 & 345 & 58.4 & $\Lambda-3.403$ & 0.002 \\
\hline Doing exercise & & & & & & \\
\hline Yes & 27 & 17.6 & 123 & 20.8 & $\mathrm{x}^{2}-0.756$ & \\
\hline No & 126 & 82.4 & 468 & 79.2 & $X^{2}=0.756$ & 0.384 \\
\hline
\end{tabular}

$\chi^{2}, \mathrm{p}: \chi^{2}$ and $\mathrm{p}$ values for Chi square test, *: Statistically significant at $\mathrm{p} \leq 0.05$.

Table 5. Correlation between percent score of barrier of health promotion and women characteristics

\begin{tabular}{|l|c|c|}
\hline \multicolumn{3}{|c|}{ \% score of barrier of health promotion } \\
\hline Variables & 0.006 & $\mathbf{r}_{\mathbf{s}}$ \\
\hline Age & $0.158^{*}$ & $<.862$ \\
\hline BMI & $-0.205^{*}$ & $<0.001^{*}$ \\
\hline Income & $0.200^{*}$ & $<0.001^{*}$ \\
\hline Level of education & $0.136^{*}$ & $<0.001^{*}$ \\
\hline Duration of diseases & $-0.148^{*}$ & $<0.001^{*}$ \\
\hline Women' impression of their health & -0.068 & $<0.001^{*}$ \\
\hline Coffee and tea taking per day & 0.007 & 0.066 \\
\hline Smoking & & 0.858 \\
\hline
\end{tabular}

$\mathrm{r}_{\mathrm{s}}$ : Spearman coefficient

*: Statistically significant at $\mathrm{p} \leq 0.05$. 
Table 6. Multivariate analysis binary logistic regression affecting barrier of health promotion

\begin{tabular}{|c|c|c|c|c|c|}
\hline \multirow{2}{*}{ Variables } & \multirow{2}{*}{ B } & \multirow{2}{*}{ Sig. } & \multirow{2}{*}{ OR } & \multicolumn{2}{|c|}{$95 \%$ CI } \\
\hline & & & & $\mathbf{L L}$ & UL \\
\hline Age & -0.398 & $0.002^{*}$ & 0.672 & 0.524 & 0.862 \\
\hline BMI & 0.755 & $<0.001^{*}$ & 2.127 & 1.525 & 2.966 \\
\hline Occupation & -0.758 & $<0.001^{*}$ & 0.468 & 0.323 & 0.680 \\
\hline Income & -1.282 & $<0.001^{*}$ & 0.277 & 0.158 & 0.486 \\
\hline Level of education & -0.150 & 0.275 & 0.861 & 0.658 & 1.127 \\
\hline Types of chronic diseases & 0.126 & $0.017^{*}$ & 1.135 & 1.023 & 1.258 \\
\hline Duration of diseases & 0.240 & $0.019^{*}$ & 1.271 & 1.041 & 1.552 \\
\hline Support from family/friends & -1.070 & $0.003^{*}$ & 0.343 & 0.168 & 0.701 \\
\hline Women' impression of their health & -0.237 & 0.106 & 0.789 & 0.592 & 1.051 \\
\hline Periodic medical checkup & -2.106 & $<0.001^{*}$ & 0.122 & 0.057 & 0.258 \\
\hline
\end{tabular}

B: Unstandardized Coefficients

OR: Odds ratio

CI: Confidence interval

LL: Lower limit

UL: Upper Limit.

\section{Discussion}

Exploring the barriers to health promotion for women with chronic diseases help to prevent and reduce disease complications and thus contribute to improving the level of health in the community by providing godliness regarding the important of health promotion activities based on individual's needs. Because there is insufficient data on the health behaviors of Egyptian women with chronic diseases and determined health promotion barriers, the current study aims to assess the barriers to health promotion activities through perception of women with chronic disease.

The current study showed that the majority of studied women had a high level of health promotion barriers. This could be because the multi-responsibility of the women in Egypt and this is also the result of the sacrifice made by most mothers. The finding is constituted with (Becker \& Stuifergin, 2004) [20] who found that adult with disability had a high level of health promotion barriers.

As regarding to the highest ranking in health promotion barriers, the current study revealed that the first barrier through the research sample suffered from embarrassment of the general appearance, especially obese women. This negatively affects their self-confidence and interest in their health. This finding is consistent with Bird et al., 2009; Crane \& Mcsweeney, 2003; Newsson \& Kemps, 2007) Results also showed that the second ranking of the Health Promotion Barrier was due to insufficient family support for women in the research sample while the third barrier was lack of help them from the health team. This reflects deficiency a culture concerning the concept of prevention better than treatment either in the research sample or within the health team, which emphasizes the importance of spreading the culture of health promotion within the Egyptian society. [21, 22, 23]

In the same table, it showed that the lowest barriers to the research sample based on their perception concern about safety, lack of transport and overlapping responsibilities. This finding reflects the fact that the real barrier to health promotion is the negative perception of the study sample regarding the importance of promoting health as a line of defense to prevent the complications of their chronic diseases that need to change to a positive vision through increase health guidelines. These results are consistent with many studies which confirmed that the dissemination of the culture of health promotion contributes to improving the health status of patients and the prevention of complications of disease. (Crane \& McSweeney, 2003; Newson \& Kemps, 2007; Wilcox et al., 2005). [22,23,24]

In contrast to other studies of barriers in women, which stated that overlapping responsibilities in women are important barriers as the presence of many responsibilities reduce their concern for their health. $[25,26,27,28]$

The current study revealed that a highly significant association was detected between the levels of Health Promotion Barriers and type of chronic disease. The current finding discovered that the lowest Score of health promotion barriers has been linked to C.O.P.D and thyroid disease, and this result can be interpreted by these two types of disease that have a less physical effect than allowing the patient to follow-up and self-care as well as the resulting complications a long time to occur. This interpretation is supported by the same results of study conducted by Smith 2013. [29]

At the same table, it was noted that the diseases of diabetes, blood pressure and rheumatoid have the highest health promotion barriers, this result may be due to fact that these diseases have signs and symptoms that to directly affect the patient's physical condition, such as diabetic coma, severe headache caused by blood pressure and also rheumatoid arthritis pain. This explanation has been confirmed through several studies, which have focused on the relationship between chronic diseases prevention and women's health promotion. Schmidt, (2016) and Vogeli, (2007). [7,30] In addition, the culture of Egyptian women in their priorities is caring for her family at the expense of their health.

The present study findings revealed a significant positive association between the levels of Health Promotion Barriers and body mass index. These results 
are logical because there is a strong relationship between obesity or weight gain and chronic diseases. These results are logical because there is a strong relationship between obesity or weight gain and chronic diseases. These findings go conversely with results in the study of Crane \& McSweeney, 2003 and Perry et al. 2008. [22,32]

The present study findings revealed a highly significant association between income and levels of Health Promotion Barriers which is not surprising given many participants, reported a not sufficient income. These findings go conversely with results in the study of Kharicha et al., 2007. [33]

\section{Conclusion}

Chronic diseases are common health problem, not only for individuals, but also for communities and governments at all levels. This study contributes to developing some understanding of perception of women with chronic diseases about the barriers to action that women face. This study concludes that: women with chronic diseases had a high score of health promotion' barriers. And there is a strong significant correlation between the levels of Health Promotion Barriers' and body mass index, level of education and duration of chronic disease.

\section{Recommendation}

The study recommends a greater emphasis on assessing and managing perceived barriers to health promotion activities in health education and policy development and proposes a conceptual model for understanding perceived barriers to action.

\section{Limitations}

There are several limitations to this study. Firstly, recruitment affected by time constraints, Secondly, the homogenous socio-demographic characteristics of the participants, who were recruited from primary health care centers and outpatient clinics of governmental hospital located in Port-Said City means that the sample is not necessarily representative of the wider population of women with chronic disease. Finally, the data analyzed in this study were collected from a self-report questionnaire which may have introduced information bias, where participants over or under-reported weight, lifestyle factors and barriers. These factors may limit the extent to which these results can be generalized to wider population of women with chronic disease.

\section{References}

[1] WHO. 2002 Innovative care for chronic conditions: building blocks for action. Geneva: World Health Organization; http://applications.emro.who.int/dsaf/EMROPUB_2016_EN_1916 9.pdf?ua=1 accessed 27 January 2018.

[2] Quality of Life in Chronic Disease Patients. Kalliopi Megari Author information Articl Health Psychol Res. 2013 Sep 24; 1(3): e27. Published online 2013 Sep 23.
[3] Medicine Net.com 2018) Medical Definition of Chronic disease. https://www.medicinenet.com/script/main/art.asp?articlekey=3349 0. accessed 27 January 2018.

[4] World health organization (2017) Eastern Mediterranean Region Framework for health information systems and core indicators for monitoring health situation and health system performance Country statistical profiles. CC BY-NC-SA 3.0 IGO licence. http://applications.emro.who.int/dsaf/EMROPUB_2016_EN_1916 9.pdf?ua=1 accessed 12 January 2018).

[5] Global health estimates 2014 summary tables: Estimated deaths by cause, sex and WHO Member State 2012. Geneva: World Health Organization; 2014

http://www.who.int/healthinfo/global_burden_disease/ estimates/en/index1.html, accessed 12 January 2018.

[6] Abbott, P., Davison, J., Moore, L., \& Rubintein, R. (2010). Barriers and enhancers to dietary behaviour change for Aboriginal people attending a diabetes cooking course. Health Promotion Journal of Australia, 21(1).

[7] Schmidt H. (2016): Chronic disease prevention and health promotion. NCBI Bookshelf. University of Pennsylvania, Philadelphia, PA, USA. April 13, 2016.

[8] American Heart Association. (2013). Herat disease and stroke statistics- http://www.ncbi.nlm.nih.gov/book/NBK435776.

[9] Chew L, Cheah C, Koh YH. Health promotion program in the private workplaces in Singapore: a prevalence survey. Singapore Med J 2002; 43: 18-24.

[10] Kroneman M, Boerma W, van den Berg M, Groenewegen P, de Jong J, van Ginneken E (2016). The Netherlands: health system review. Health Systems in Transition, 2016; 18(2):1-239.

[11] Daly G (2004). How a health promotion model reduces disabling complications of diabetes at https://www.nursingtimes.net/clinical-archive/diabetes/how-ahealth-promotion-model-reduces-disabling-complications-of diabetes/199689. article accessed 12 January 2018).

[12] El Nouman A., El Derwi D., Abdel Hai Rand Abou Zeina H. (2009). Female youth health promotion model in primary health care: a community-based study in rural Upper Egypt. Eastern Mediterranean Health. Journal Department of Public Health and Community Medicine, Faculty of Medicine, University of Cairo, Cairo, EMHJ, 2009, 15(6):1513-1524.

[13] Kronfol N.M. Access and barriers to health care delivery in Arab countries: a review Eastern Mediterranean Health Journal. Lebanese Health Care Management Association, Beirut, Lebanon. EMHJ, 2012, 18(12):1239-1246.

[14] Stanton W, Balanda K, Gillespie A \& Lowe J. Barriers to health promotion activities in public hospitals Article in Australian and New Zealand Journal of Public Health 20 (5): 500-4 November 2002.

[15] Strömberg A., Mårtensson J., Fridlund B., Levin L. Å., Karlsson J. E., Dahlström U.. Nurse-led heart failure clinics improve survival and self-care behavior in patients with heart failure. Results from a prospective, randomised trial, European Heart Journal, 2003, vol. 24 (pg. 1014-1023).

[16] Griffiths C., Miles K., Aldam D., Cornforth D., Milton J., Edwards S., Williams I., A nurse- and pharmacist-led treatment advice clinic for patients attending an HIV outpatient clinic, Journal of Advanced Nursing, 2007, vol. 58 (pg. 320-326).

[17] Bosch-Capblanc X., Abba K., Prictor M., Garner P.. Contracts between patients and healthcare practitioners for improving patients' adherence to treatment, prevention and health promotion activities, The Cochrane Database of Systematic Reviews, 2009, vol. 2007 (pg. 1-73) The Cochrane Collaboration. Published by John Wiley \& Sons, Ltd.

[18] Keleher H., Parker R., Abdulwadud O., Francis K. (2009). Systematic review of the effectiveness of primary care nursing, International Journal of Nursing Practice , 2009, vol. 15 (pg. 16-24).

[19] WHO. Obesity. Preventing and managing the global epidemic. (1998) Report of WHO Consuitation on Obesity, Geneva, World Health Organization, 1998.

[20] Becker, H. \& Stuifbergen, A.K. (2004). What makes it so hard? Barriers to health promotion experienced by people with multiple sclerosis and polio. Family and Community Health 27(1), 75-83.

[21] Bird, S., Radermacher, H., Feldman, S., Sims, J., Kurowski, W., Brownings, C., (2009). Factors influencing the phys-ical activity levels of older people from culturally diverse communities: An Australian experience. Ageing \& Society, 29, 1275-1294. 
[22] Crane, P. B., \& McSweeney, J. C. (2003). Exploring older women's lifestyle changes after myocardial infarction. Medsurg Nursing: Official Journal of The Academy of Medical-Surgical Nurses, 12(3), 170-176.

[23] Newson, R. S., \& Kemps, E. B. (2007). Factors that promote and prevent exercise engagement in older adults. Journal of Aging and Health, 19(3), 470-481.

[24] Wilcox, S., Oberrecht, L., Bopp, M., Kammermann, S. K., \& McEl-murray, C. T. (2005). A qualitative study of exercise in older African American and white women in rural South Carolina: Perceptions, barriers, and motivations. Journal of Women \& Aging, 17(1-2), 37-53.

[25] Bowen, D. J., Balsam, K. F., Diergaarde, B., Russo, M., \& Escamilla, G. M. (2006). Healthy eating, exercise, and weight: Impressions of sexual minority women. Women \& Health, 44(1), 79-93.

[26] Juarbe, T., Turok, X. P., \& Perez-Stable, E. J. (2002). Perceived benefits and barriers to physical activity among older Latina women. Western Journal of Nursing Research, 24(8), 868-886.

[27] Osuji, T., Lovegreen, S. L., Elliott, M., \& Brownson, R. C. (2006) Barriers to physical activity among women in the rural midwest. Women \& Health, 44(1), 41-55.
[28] Ansari, W. E., \& Lovell, G. (2009). Barriers to exercise in younger and older non-exercising adult women: A cross sectional study in Lon-don, United Kingdom. International Journal Of Environmental Research And Public Health, 6, 1443-1455.

[29] Smith M., Ory M., Ahn S., and Miles T. (2013). Factors associated with women's chronic disease management: Associates of healthcare frustrations, physician support and self-care needs. J Aging Res. Oct.; 2.

[30] McGuire, Amanda Mary (2011). Factors influencing health promotion activities in midlife and older Australian women with a chronic disease. Masters by Research thesis, Queensland University of Technology.

[31] Vogeli C., Shields AE., \& Lee TA, et al. Multiple chronic conditions: prevalence, health consequences, and implications for quality care management, and costs. Journal of General Internal Medicine.2007; 22(3):391-395.

[32] Perry, C. K., Rosenfeld, A. G., \& Kendall, J. (2008). Rural women walking for health. Western Journal of Nursing Research, 30(3), 295-316.

[33] Kharicha, K., Iliffe, S., Harari, D., Swift, C., Gillman, G., \& Stuck, A. E. (2007, April). Health risk appraisal in older people 1: Are older people living alone an 'at risk' group? British Journal of General Practice, 271-276. 